\title{
PHOTOMETRIC VARIABILITY IN A WARM, STRONGLY MAGNETIC DQ WHITE DWARF, SDSS J103655.39+652252.2
}

\author{
Kurtis A. Williams ${ }^{1}$, D. E. Winget ${ }^{2}$, M. H. Montgomery ${ }^{2}$, Patrick Dufour ${ }^{3}$, S. O. Kepler ${ }^{4}$, J. J. Hermes ${ }^{2}$, \\ Ross E. Falcon ${ }^{2}$, K. I. WingeT ${ }^{2}$, Michael Bolte ${ }^{5}$, Kate H. R. Rubin ${ }^{6}$, and James Liebert ${ }^{7}$ \\ ${ }^{1}$ Department of Physics \& Astronomy, Texas A\&M University-Commerce, P.O. Box 3011, Commerce, TX 75429, USA; Kurtis.Williams@tamuc.edu \\ ${ }^{2}$ Department of Astronomy, University of Texas, 1 University Station C1400, Austin, TX 78712, USA \\ ${ }^{3}$ Département de Physique, Université de Montréal, C.P. 6128, Succ. Centre-Ville, Montréal, QC H3C 3J7, Canada \\ ${ }^{4}$ Departamento de Astronomia, Universidade Federal do Rio Grande do Sul, Av. Bento Gonçalves 9500 Porto Alegre 91501-970, RS, Brazil \\ ${ }^{5}$ UCO/Lick Observatory, University of California, 1156 High St., Santa Cruz, CA 95064, USA \\ ${ }^{6}$ Max-Planck-Institut für Astronomie, Königstuhl 17, D-69117 Heidelberg, Germany \\ Received 2012 August 1; accepted 2013 April 10; published 2013 May 14
}

\begin{abstract}
We present the discovery of photometric variability in the DQ white dwarf SDSS J103655.39+652252.2 (SDSS J1036+6522). Time-series photometry reveals a coherent monoperiodic modulation at a period of $1115.64751(67) \mathrm{s}$ with an amplitude $0.442 \% \pm 0.024 \%$; no other periodic modulations are observed with amplitudes $\gtrsim 0.13 \%$. The period, amplitude, and phase of this modulation are constant within errors over 16 months. The spectrum of SDSS J1036+6522 shows magnetic splitting of carbon lines, and we use Paschen-Back formalism to develop a grid of model atmospheres for mixed carbon and helium atmospheres. Our models, while reliant on several simplistic assumptions, nevertheless match the major spectral and photometric properties of the star with a self-consistent set of parameters: $T_{\text {eff }} \approx 15,500 \mathrm{~K}, \log g \approx 9, \log (\mathrm{C} / \mathrm{He})=-1.0$, and a mean magnetic field strength of $3.0 \pm 0.2 \mathrm{MG}$. The temperature and abundances strongly suggest that SDSS J1036+6522 is a transition object between the hot, carbon-dominated DQs and the cool, heliumdominated DQs. The variability of SDSS J1036+6522 has characteristics similar to those of the variable hot carbon-atmosphere white dwarfs (DQVs), however, its temperature is significantly cooler. The pulse profile of SDSS J1036+6522 is nearly sinusoidal, in contrast with the significantly asymmetric pulse shapes of the known magnetic DQVs. If the variability in SDSS J1036+6522 is due to the same mechanism as other DQVs, then the pulse shape is not a definitive diagnostic on the absence of a strong magnetic field in DQVs. It remains unclear whether the root cause of the variability in SDSS J1036+6522 and the other hot DQVs is the same.
\end{abstract}

Key words: stars: evolution - stars: individual (SDSS J103655.39+652252.2) - stars:

magnetic field - stars: oscillations (including pulsations) - white dwarfs

Online-only material: color figures

\section{INTRODUCTION}

White dwarfs (WDs), the final stage of stellar evolution for the vast majority of stars, exhibit a menagerie of spectral characteristics. This is somewhat surprising at first, as the extreme surface gravities of WDs should chemically stratify their atmospheres on astronomically short timescales (e.g., Dupuis et al. 1992; Zuckerman et al. 2003). The origins of the spectral species of WDs are thought to be either a result of the final stages of stellar evolution, such as a late thermal pulse exhausting the residual hydrogen in a star (e.g., Iben et al. 1983), or as a result of physical processes along the WD cooling track, such as convective mixing and accretion (e.g., Koester 1976; Dupuis et al. 1993; Tremblay \& Bergeron 2008). An understanding of these origins is crucial for many areas of astrophysical interest, such as the cooling rates (and therefore age-dating) of WD populations (e.g., Winget et al. 1987; Hansen et al. 2004; von Hippel 2005), the fates of planetary systems (e.g., Jura 2003; Zuckerman et al. 2007; Farihi et al. 2009), and physical processes during the post main-sequence evolution of stars (e.g., Weidemann 1990; Hansen 1999; Fontaine et al. 2001; Winget \& Kepler 2008).

\footnotetext{
7 Emeritus, Steward Observatory, University of Arizona, 933 N. Cherry Ave., Tucson, AZ 85721, USA; jamesliebert@ gmail.com
}

Using spectral data from the Sloan Digital Sky Survey (SDSS), Liebert et al. (2003) identified 18 WDs of spectral class DQ whose spectra exhibit lines of neutral and/or ionized atomic carbon, while the majority of DQ WDs have spectral features indicative of molecular carbon, such as Swan bands. A few years later, another DQ WD with features of atomic carbon was discovered in the field of the young open star cluster Messier 35 (Williams et al. 2006). Both papers suggest that these WDs had helium-dominated atmospheres with traces of carbon sufficient to mask the helium spectral features. Shortly thereafter, Dufour et al. $(2007,2008$ a) recognized that, instead, these "hot DQ" WDs $\left(T_{\text {eff }} \approx 18,000-24,000 \mathrm{~K}\right)$ have carbon-dominated atmospheres, with carbon abundances $\log (\mathrm{C} / \mathrm{He}) \gtrsim 1$.

The origin of these carbon-dominated atmospheres in a relatively small temperature range is difficult to explain. Dufour et al. (2008a) propose a spectral evolution scenario, expanded upon by evolutionary calculations of Althaus et al. (2009). In this scenario, a hot WD undergoes a vigorous very late thermal pulse that reduces the residual He content of the star to $\sim 10^{-8}-10^{-7} M_{\mathrm{WD}}$. Immediately following the very late thermal pulse, the WD would look like the very hot, carbon/oxygenrich WD H1504+65 (e.g., Werner et al. 2004). The residual helium would then rise to the surface of the WD, causing it to evolve to spectral type DB (helium-atmosphere), until a convective zone mixes this helium veneer back into the carbon-rich 
lower atmosphere at roughly the temperature where the hot DQs appear.

Alternatively, hot DQ WDs could be remnants of the socalled "super-AGB" stars, stars massive enough to ignite carbon, resulting in an oxygen-neon WD surrounded by a layer of carbon and oxygen; Garcia-Berro et al. (1997) predict that such a star could have no residual hydrogen and little, if any, residual helium. This latter scenario requires that all hot DQ WDs should be massive $\left(\gtrsim 1 M_{\odot}\right)$, while the former scenario allows for lowermass hot DQ stars.

WD asteroseismology is a well-established method for studying the parameters and interior structures of WDs, provided non-radial pulsations are present and can be detected. Simple arguments involving the thermal timescale at the base of the convection zone led Montgomery et al. (2008) to suspect that some hot DQs might be unstable to non-radial pulsations, and observations inspired by this possibility led to the discovery of variations in the hot DQ SDSS J142625.70+565218.4 (hereafter SDSS J1426+5652). Nearly simultaneous with the publication of this discovery, Fontaine et al. (2008) published a prediction based on detailed nonadiabatic calculations that hot DQs may be unstable to nonradial pulsations if sufficient helium is present in the atmosphere. Subsequently, Córsico et al. (2009) published nonadiabatic pulsation analysis of hot DQs based on their hot DQ evolutionary models. Crucially, all three of these pulsational stability analyses present significantly different predictions of the location of the hot DQ instability strip in $T_{\text {eff }}$ and $\log g$ space.

Observations of variable hot DQs have uncovered additional mysteries about these stars. The folded pulse shape of SDSS J1426+5752 differs significantly from those of pulsating DA and DB stars (Montgomery et al. 2008), and the star's spectrum shows Zeeman splitting indicative of a strong magnetic field ( 1.2 MG; Dufour et al. 2008b). Following the discovery of variability in SDSS J1426+5752, Barlow et al. (2008) detected variability in two additional hot DQ stars, SDSS J220029.08-073121.5 (hereafter SDSS J2200-0741) and SDSS J234843.30-094245.3 (hereafter SDSS J2348-0942). They claim both variable hot DQs have pulse shapes similar to SDSS J1426+5752. Additional observations of these two stars by Dufour et al. (2009a) agree with Barlow et al. (2008) that SDSS J2200-0741 has a pulse shape similar to SDSS J1426+5752, but they find that SDSS J2348-0942 has a pulse shape more typical of other classes of pulsating WDs. This, combined with suggestions of Zeeman splitting in the spectrum of SDSS J2200-0741, leads Dufour et al. (2009a) to suggest that SDSS J2200-0741 also has a strong magnetic field, while SDSS J2348-0942 does not.

Dunlap et al. (2010) announced the discovery of the DQV, SDSS J133710.19-002643.5 (hereafter SDSS J1337-0026). This star has low-amplitude $(\sim 0.3 \%)$ photometric variations and has a strongly non-sinusoidal pulse shape (Dunlap et al. 2010). While their low-resolution, high signal-to-noise spectrum shows no evidence for Zeeman splitting, a higher-resolution spectrum shows clear Zeeman splitting (P. Dufour, in preparation). Could this star have a magnetic field strong enough to distort the pulse shape (by whatever physical mechanism), yet be weak enough to not cause noticeable Zeeman splitting in low-resolution spectra or is there no relation between magnetism and the pulse shape?

More recently, Dufour et al. (2011a) discovered variability in yet another hot DQ, WD 1150+012 (SDSS J1153+0056), based on variable photon flux rates in the FUV spectrum obtained by the Cosmic Origins Spectrograph on the Hubble Space
Telescope. These observations were not long enough to establish a pulse shape for this object.

Most of these studies have been conducted under the presumption that the variability in DQVs is due to nonradial pulsations. In addition to initial models suggesting that DQVs may be unstable to such pulsations (Montgomery et al. 2008; Fontaine et al. 2008), claims have been made of the detection of additional modes of variability not harmonically related to the dominant mode (Dufour et al. 2009a; Green et al. 2009). If confirmed, these additional modes would be evidence in favor of pulsations. However, other mechanisms for variability have been postulated, including the musings of Montgomery et al. (2008) of accretion of carbon-rich material in an AM CVnlike system and the hypothesis floated by Dufour et al. (2008b) that the oscillations could be analogous to those observed in rapidly-oscillating Ap stars. Other potential causes could include short-period binary orbits or rapid rotation of these WDs. We discuss these possibilities more thoroughly in Section 4.

The growing number of variable hot DQ WDs should help to address many of the conundrums surrounding these objects. In particular, mapping the hot DQ instability strip can test the three independent predictions of pulsational properties and stability. Additional pulse profiles can test whether the pulse shapes of variable hot DQs are influenced by the presence or absence of a magnetic field. Finally, additional variables will help to define further the emerging characteristics of this enigmatic class of variable star.

In this paper, we announce the discovery of variability in another DQ star, SDSS J103655.39+652252.2, ${ }^{8}$ hereafter SDSS J1036+6522. SDSS J1036+6522 was identified by Liebert et al. (2003) as a WD with absorption lines of atomic carbon. Schmidt et al. (2003) identify three sets of Zeeman-split C I lines in the star's spectrum; the splitting implies a mean field strength of $2.9 \mathrm{MG}$, or a dipole field strength of $\sim 4 \mathrm{MG}$. Our spectral analysis (Section 3) finds that SDSS J1036+6522 is significantly cooler than any of the other known DQVs. In Section 4 we discuss the potential implications of this discovery for the definition and characteristics of DQV WDs.

\section{TIME-SERIES PHOTOMETRY AND ANALYSIS}

Positional and photometric parameters for SDSS J1036+6522 are taken from the SDSS Data Release 8 (Abazajian et al. 2009). SDSS J1036+6522 is located at R.A. $=10^{\mathrm{h}} 36^{\mathrm{m}} 55^{\mathrm{s}} .39$, decl. $=+65^{\circ} 22^{\prime} 52^{\prime \prime}$. 2 (J2000). The star's photometric properties via SDSS point-spread function photometry are $g=18.514 \pm$ $0.024, u-g=-0.217 \pm 0.032$, and $g-r=-0.325 \pm 0.031$. The star has a significant proper motion, $\mu_{\alpha}=26 \pm 1 \mathrm{mas} \mathrm{yr}^{-1}$;

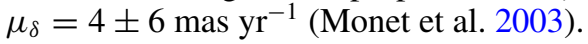

SDSS J1036+6522 was included in the Liebert et al. (2003) catalog of SDSS WDs with spectral features of atomic carbon. At the time of our first observing run, the spectral parameters $T_{\text {eff }}$ and $\log g$ were unknown. The star's selection was motivated by its very blue colors, which suggested it might be of similar temperature to the other known DQVs.

Time-series photometry of SDSS J1036+6522 was obtained over several nights in late 2008, early 2009, and early 2010 with the Argos high-speed photometer on the McDonald Observatory $2.1 \mathrm{~m}$ Otto Struve Telescope (Nather \& Mukadam 2004). All observations were taken through a $1 \mathrm{~mm}$ Schott glass BG40 filter. A log of observations is presented in Table 1.

\footnotetext{
8 a.k.a. SDSS J103655.38+652252.1 in Liebert et al. (2003) and SDSS J103655.38+652252.0 in Eisenstein et al. (2006).
} 

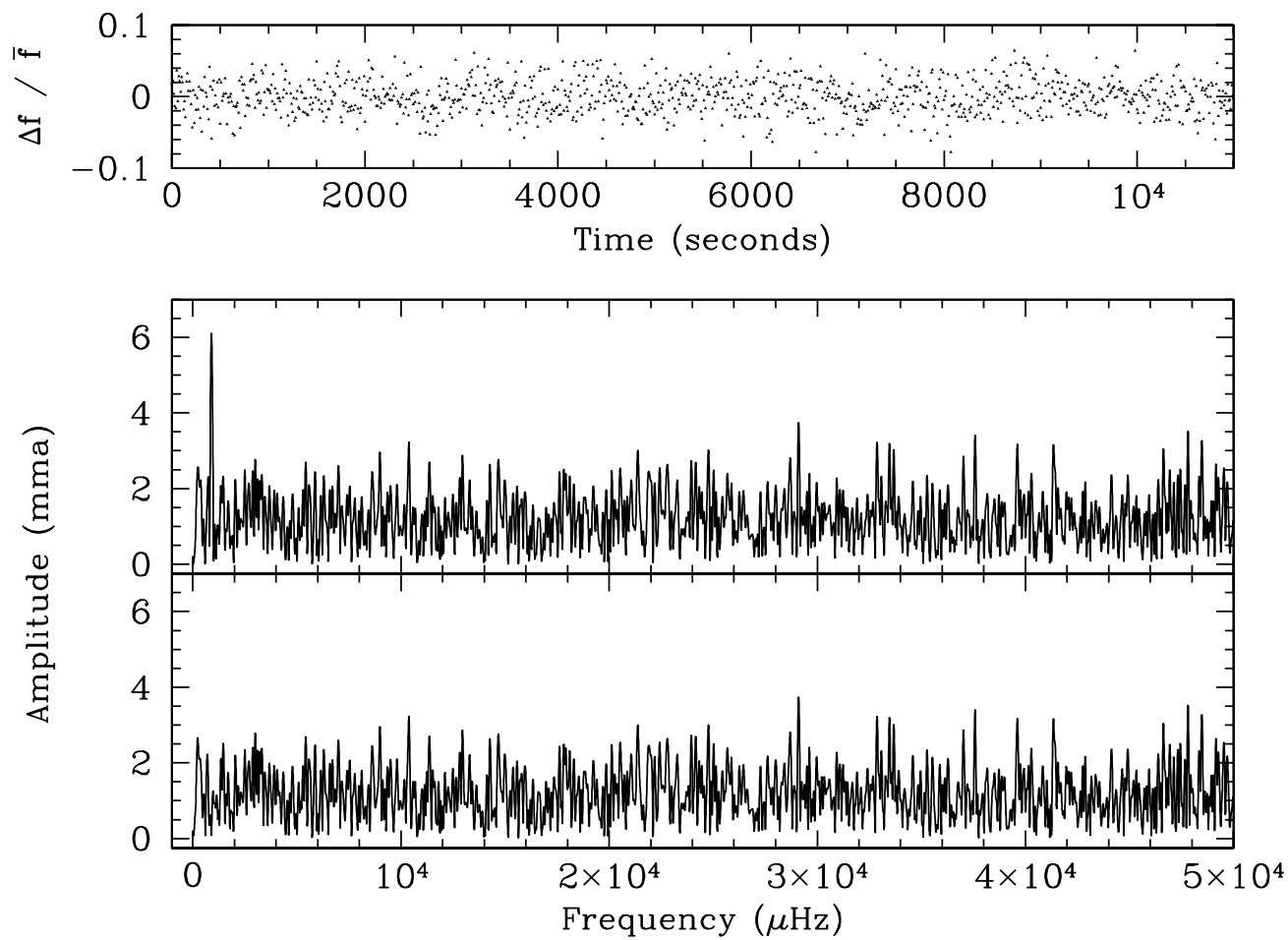

Figure 1. Time-series photometry and analysis from the discovery run of SDSS J1036+6522. Top panel: photometry from $10 \mathrm{~s}$ exposures, plotted in terms of fractional amplitude $f$. No significant modulation is obvious. Middle panel: discrete Fourier transform of the data in the top panel. A significant signal with amplitude $6.2 \mathrm{mma}$ is seen at $897.3 \mu \mathrm{Hz}$. Bottom panel: discrete Fourier transform after pre-whitening the photometric data by the $897.3 \mu \mathrm{Hz}$ signal. No other significant peaks are present.

Table 1

Observation Log

\begin{tabular}{lccccc}
\hline \hline UT Date & $\begin{array}{c}\text { Run } \\
\text { Name }\end{array}$ & $\begin{array}{c}\text { Start } \\
\left(\text { BJD }_{\text {TDB }}\right)\end{array}$ & $\begin{array}{c}\text { Exp. Time } \\
(\mathrm{s})\end{array}$ & $\begin{array}{c}\text { Run Length } \\
(\mathrm{s})\end{array}$ & $\begin{array}{c}\text { Seeing } \\
(\operatorname{arcsec})\end{array}$ \\
\hline 2008 Dec 29 & A1807 & 2454829.88044726 & 10 & 11000 & 1.4 \\
2008 Dec 31 & A1811 & 2454831.79331520 & 30 & 7560 & 1.8 \\
2009 Jan 2 & A1816 & 2454833.80946591 & 30 & 20430 & 2.6 \\
2009 Jan 3 & A1820 & 2454834.91942706 & 30 & 11130 & 1.6 \\
2009 Jan 4 & A1822 & 2454835.78627879 & 30 & 22530 & 1.3 \\
2009 Apr 21 & A1858 & 2454942.64579972 & 15 & 17400 & 1.1 \\
2009 Apr 22 & A1861 & 2454943.61438557 & 15 & 22455 & 1.5 \\
2009 Apr 30 & A1871 & 2454951.59850661 & 15 & 15300 & 1.5 \\
2010 Jan 16 & A2059 & 2455212.82071991 & 15 & 19035 & 2.3 \\
2010 Feb 13 & A2076 & 2455240.69756942 & 15 & 26595 & 2.2 \\
2010 Feb 14 & A2080 & 2455241.87175010 & 15 & 13758 & 1.3 \\
2010 Apr 12 & A2117 & 2455298.63301727 & 15 & 17400 & 1.9 \\
\hline
\end{tabular}

Note. Start time is midpoint of first exposure.

The photometric data were reduced with the methods and pipeline described in Mullally et al. (2005) and improved upon in Mullally et al. (2008). Weighted aperture photometry was performed using a variety of aperture radii; the final aperture radius was chosen based on the lowest noise level in the resulting discrete Fourier transform (DFT) and was typically close to the observed FWHM. The light curve was divided by a weighted combination of one to three nearby reference stars chosen to be as similar in color as possible to the target; the precise number of reference stars varied from observing run to observing run; fainter comparison stars were excluded if their light curves were of low signal-to-noise due to less-thanideal observing conditions. Differential atmospheric extinction was removed by fitting a second-order polynomial to the light curve, and sections of the light curve were excluded from further analysis if clearly affected by cloud, cosmic ray hits, obscuration by the telescope dome due to inattentive observers, etc. The data are corrected for the motion of the Earth around the solar system barycenter using the method of Stumpff (1980), and all UTC leap-seconds up to the date of observation are accounted for. No attempt was made at absolute photometric calibration.

A visual inspection of the light curves reveals no obvious signs of variability in either raw or smoothed light curves; an example light curve from the discovery run is shown in the upper panel of Figure 1. The measured standard deviation in the photometry of individual data points is $16.6 \mathrm{mma}$ ( $1 \mathrm{mma}=10^{-3}$ fractional amplitude).

In the middle panel of Figure 1 we show the DFT of the unsmoothed data from the discovery (first) observing run. A clear and significant signal is seen at a frequency of $897.3 \mu \mathrm{Hz}$ with an amplitude of $6.2 \pm 1.0 \mathrm{mma}$. The DFT of the data 


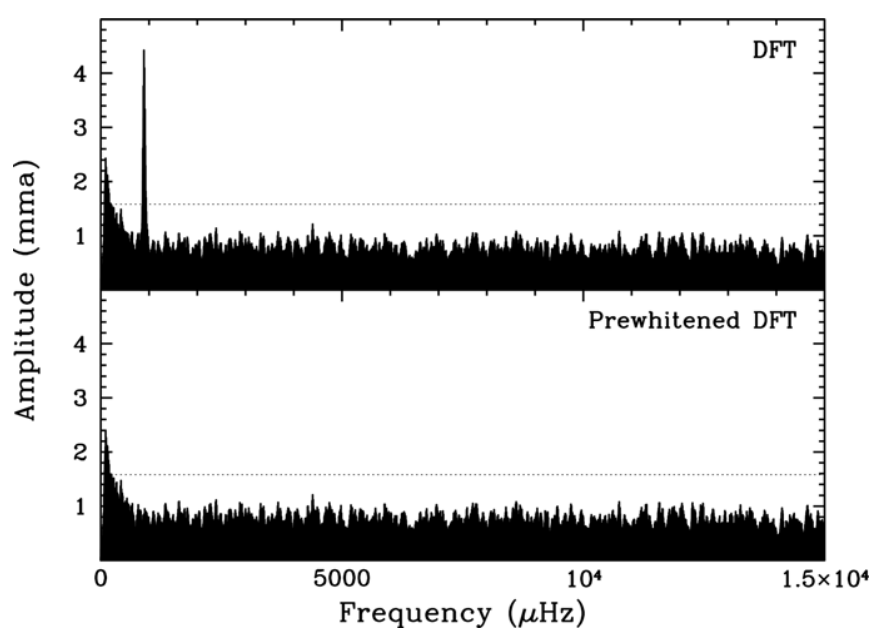

Figure 2. Top panel: the discrete Fourier transform of the combination of all time-series photometry between 2008 December and 2010 April. Bottom panel: the discrete Fourier transform of the same combined data after pre-whitening by the single significant frequency. In both panels, the horizontal dashed line indicates the $99 \%$ false-alarm probability level. Only one significant peak, the $P=1115.64751$ s modulation, is observed.

after pre-whitening by this single frequency reveals no other significant signal (bottom panel).

Combining data from multiple observing runs can reduce the noise in the DFT and permit more precise measurements of the frequency, amplitude and phase of the photometric modulation, if the modulations are coherent between each run. Our preferred methodology involves bootstrapping to determine a period precise enough to bridge multiple runs without losing a cycle count (e.g., Winget et al. 1985). Unfortunately, weather and scheduling frustrations resulted in multiple-month gaps between successful observing sequences that are too large to bridge with this method.

We therefore make the assumption that the photometric modulations are coherent across our entire data set, and we calculate a DFT for the entire combined data set; this DFT is shown in Figure 2. Expanding the plot about the primary peak, we see complex aliasing (Figure 3). The highest peak, with frequency $f=896.340458(54) \mu \mathrm{Hz}[P=1115.64751(67) \mathrm{s}]$, is the only alias consistent with the bootstrapped periods from the individual observing runs in 2008 December, ${ }^{9} 2009$ April, and 2010 January/February (i.e., the frequency of this alias is consistent with the frequency of the highest peak in each of the combined runs within calculated errors). The window function for this period is consistent with the observed aliasing, and pre-whitening the light curve by this best-fit period removes all significant structure from the DFT (Figure 3, middle and bottom panels, respectively). We therefore believe that this peak represents the true frequency of modulation.

We determine the amplitude and phase of the modulation using least-squares fitting of a sine wave to the combined data set. The resulting fit gives:

$$
A(t)=A_{0} \sin \left[2 \pi f\left(t-T_{0}\right)\right],
$$

where $A(t)$ is the observed fractional modulation, $A_{0}=4.42 \pm$ $0.24 \mathrm{mma}$ is the amplitude of the modulation, $f$ is the frequency, $t$ is the $\mathrm{BJD}_{\mathrm{TDB}}$ time of observation (Barycentric Julian Date of the Barycentric Dynamical Time, e.g., Eastman et al. 2010), and $T_{0}=$ BJD $_{\mathrm{TDB}} 2454830.00459(11)$ days.

\footnotetext{
9 We consider all data obtained between 2008 December 29 and 2009 January 4 as a single observing run.
}

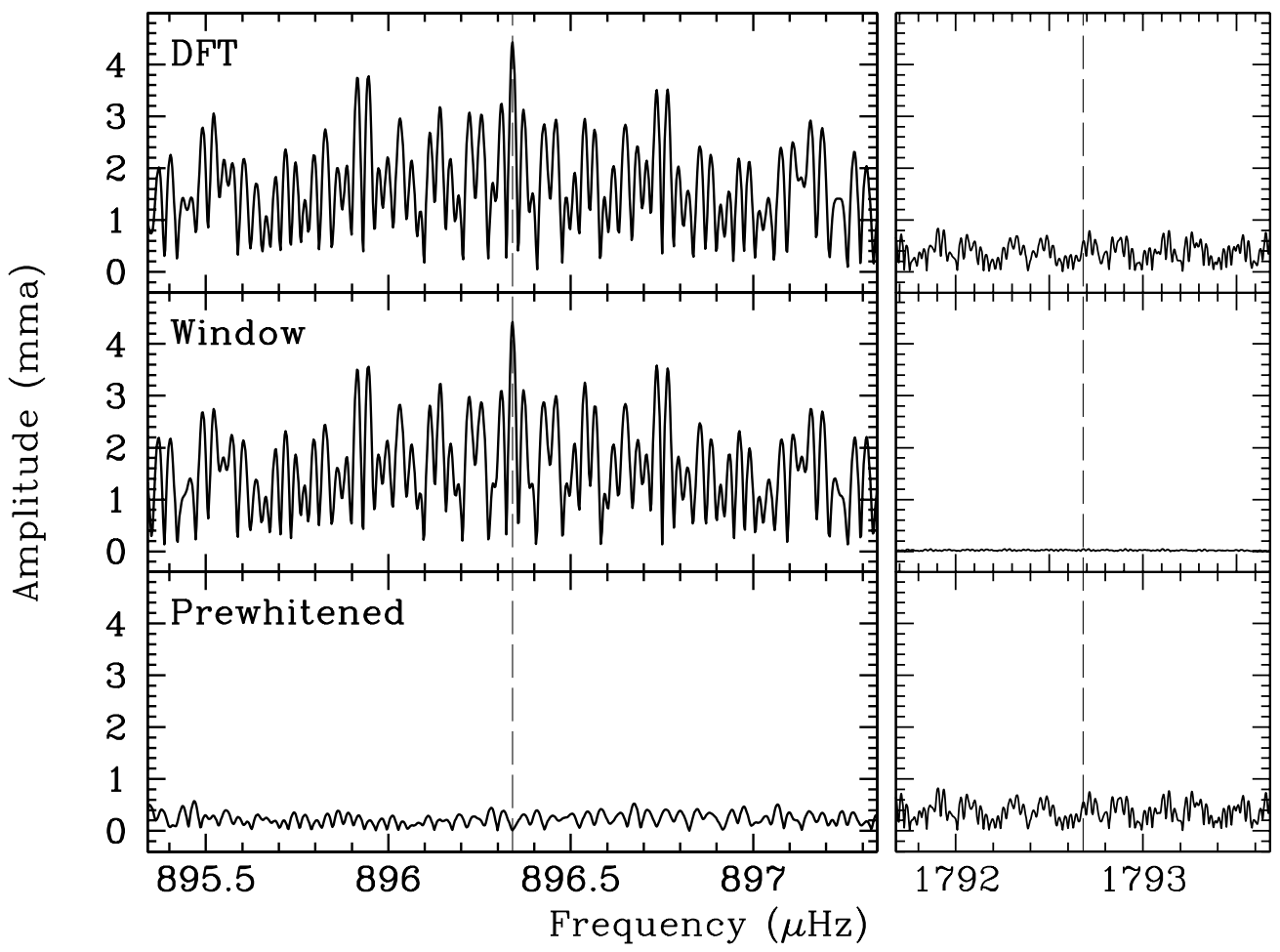

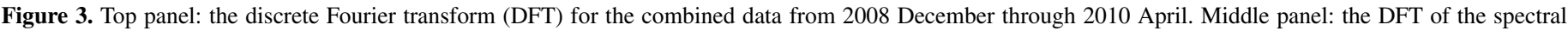

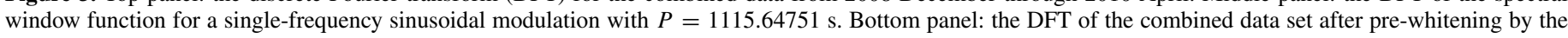

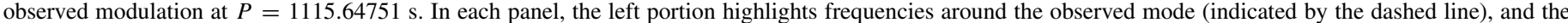

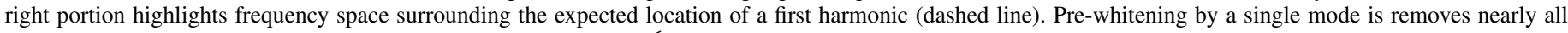
power from the DFT, and any harmonic must have an amplitude of $\lesssim 1 \mathrm{mma}$. 


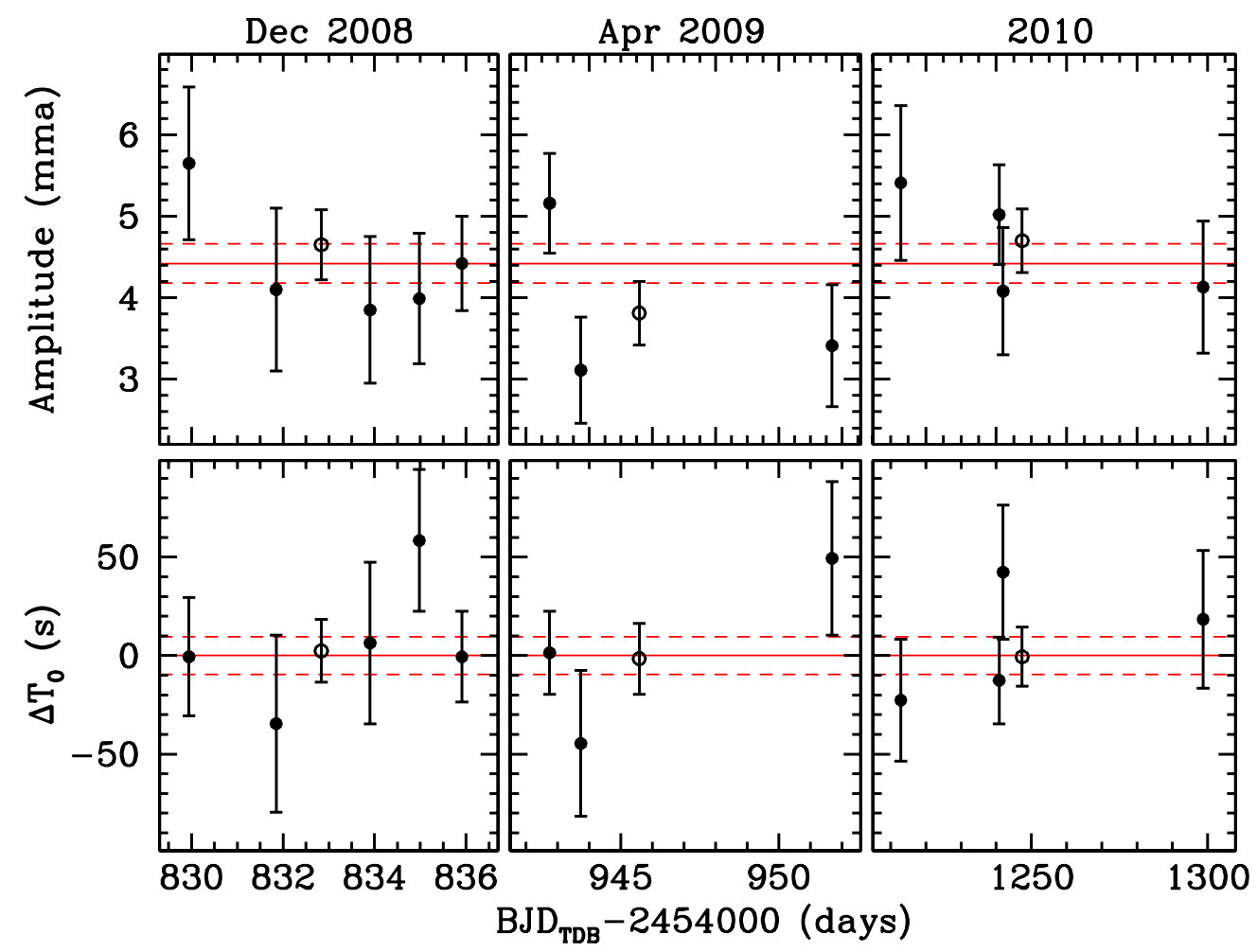

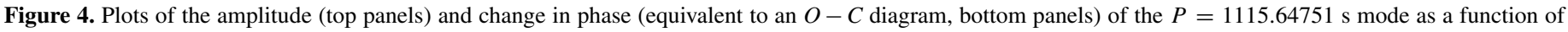

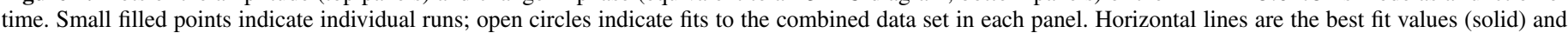

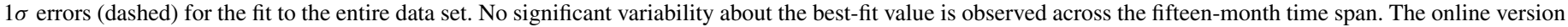
of the figure uses color for clarity.

(A color version of this figure is available in the online journal.)

Table 2

Fits for the $1115.64751 \mathrm{~s}$ Mode

\begin{tabular}{lcccc}
\hline \hline Run(s) & $\begin{array}{c}A \\
(\mathrm{mma})\end{array}$ & $\begin{array}{c}\sigma A \\
(\mathrm{mma})\end{array}$ & $\begin{array}{c}T_{0}-T_{0, \text { all }} \\
(\mathrm{s})\end{array}$ & $\begin{array}{c}\sigma T_{0} \\
(\mathrm{~s})\end{array}$ \\
\hline Entire Set & 4.42 & 0.24 & 0.0 & 9.5 \\
All 2010 & 4.70 & 0.39 & -1 & 15 \\
A11 2009 Apr & 3.81 & 0.39 & -2 & 18 \\
A11 2008 Dec & 4.65 & 0.43 & 2 & 16 \\
A1807 & 5.65 & 0.94 & -1 & 30 \\
A1811 & 4.10 & 1.00 & -35 & 45 \\
A1816 & 3.85 & 0.90 & 6 & 41 \\
A1820 & 3.99 & 0.80 & 58 & 36 \\
A1822 & 4.42 & 0.58 & -1 & 23 \\
A1858 & 5.16 & 0.61 & 1 & 21 \\
A1861 & 3.11 & 0.65 & -45 & 37 \\
A1871 & 3.41 & 0.75 & 49 & 39 \\
A2059 & 5.41 & 0.95 & -23 & 31 \\
A2076 & 5.02 & 0.61 & -13 & 22 \\
A2080 & 4.08 & 0.78 & 42 & 34 \\
A2117 & 4.13 & 0.81 & 18 & 35
\end{tabular}

Note. $T_{0}-T_{0 \text {, all }}$ is equivalent to the more familiar $O-C$ quantity.

We also use the best-fit frequency to determine amplitudes and phases for individual observing runs. These results, determined again via least-squares fitting, are given in Table 2 and plotted in Figure 4 . There is no significant variation of either the amplitude or $T_{0}$ across the 15 months, indicating that the photometric modulations were coherent and of constant amplitude over that time span. If the photometric modulations remain stable, it will be possible to constrain the rate of change of the period $(\dot{P})$ and, potentially, discriminate between various models of DQVs and their evolution.

After pre-whitening of the light curve of SDSS J1036+6522 by the best-fitting solution above, we search for additional pulsational frequencies. No other obvious peaks are seen in the DFT. We calculate false-alarm probabilities using the method of Scargle (1982) as implemented by Kepler (1993). This method gives a $99 \%$ false-alarm probability amplitude of $1.54 \mathrm{mma}$; no peaks appear in the DFT higher than this amplitude for frequencies $\gtrsim 500 \mu \mathrm{Hz}$. In particular, no harmonic is observed at a frequency of $1793 \mu \mathrm{Hz}$ (right panels of Figure 3).

As the light curve of SDSS J1036+6522 is dominated by a single frequency of modulation, we have computed a pulse shape by folding the data at the $1115.64751 \mathrm{~s}$ periodicity. The individual measurements are averaged in 100 bins based on phase, giving approximately 60 photometric points per bin. The resulting pulse shape is plotted in Figure 5 along with a sine wave of the same mean amplitude and period. Within the scatter, the pulse shape looks sinusoidal.

The bottom panel of Figure 5 shows the folded light curve after pre-whitening the data by the single observed frequency. No significant residual is seen, consistent with the interpretation of the pulse shape as a sine wave with no significant harmonics. The root-mean square of the pre-whitened data is $1.4 \mathrm{mma}$, which is consistent with predictions for the scatter in each bin assuming typical photometric errors and Poisson statistics.

\section{SPECTROSCOPIC OBSERVATIONS AND ANALYSIS}

The SDSS spectrum of SDSS J1036+6522 is of moderate signal-to-noise $(\approx 28$ per resolution element at $4600 \AA)$. 
Pulse Shape of SDSSJ1036 (no 1861)
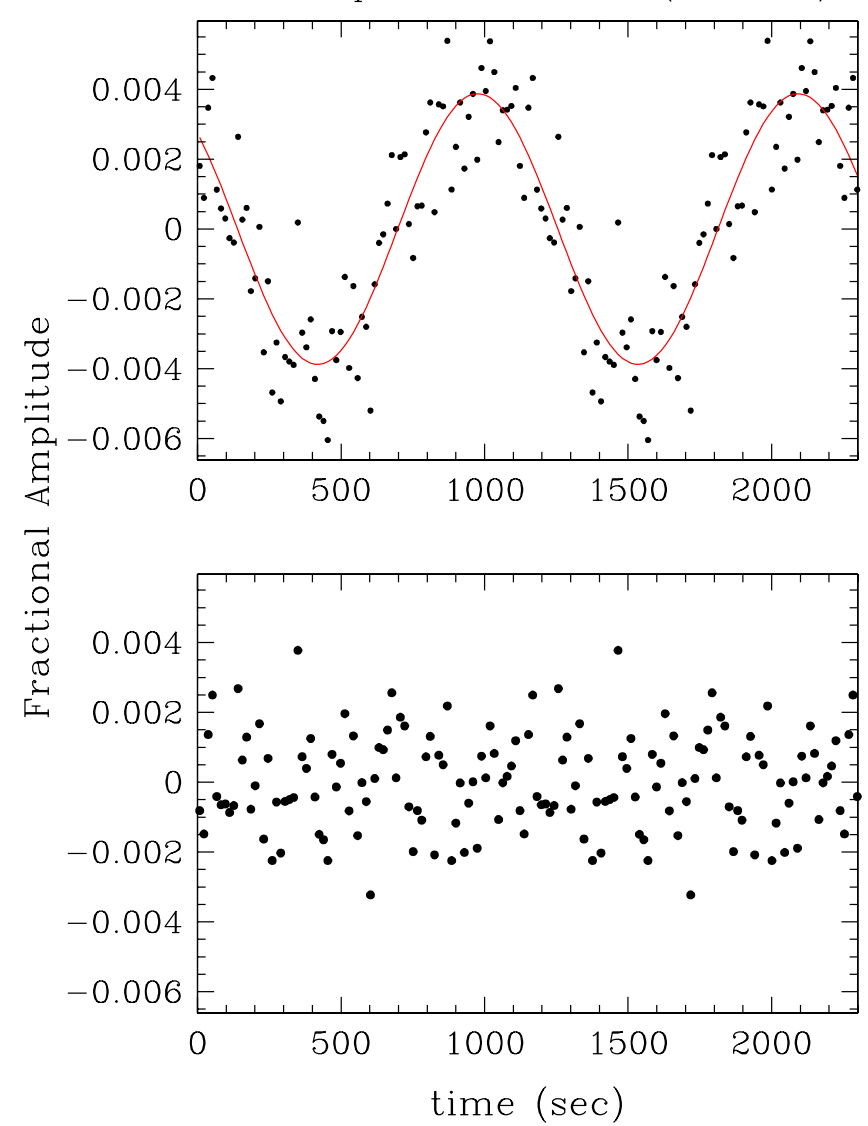

Figure 5. Top panel: the light curve of SDSS J1036+6522 folded at its sole observed period of $1115.64751 \mathrm{~s}$ (points) and a sine wave of the same mean amplitude and period (solid curve, red in the online version of the figure). Each point is the average of $\sim 60$ individual photometric measurements. Two cycles are plotted for clarity. Bottom panel: the folded light curve after pre-whitening by the single observed period. No residual signal is evident; the rms scatter in the residuals is $1.4 \mathrm{mma}$, consistent with the photometric measurement errors. The residuals show no significant structure, in agreement with the lack of significant harmonics in the DFT.

(A color version of this figure is available in the online journal.)

This was sufficient to identify the atomic carbon lines and the Zeeman splitting observed by Liebert et al. (2003) and Schmidt et al. (2003), but not to obtain precise $T_{\text {eff }}$ and $\log g$ (see Figure 6).

In order to better constrain the atmospheric parameters of SDSS J1036+6522, we obtained two sets of spectroscopic observations. We obtained three exposures at the MMT on the night of UT 2007 December 15 totaling $3600 \mathrm{~s}(3 \times 1200)$ with the Blue Channel Spectrograph. We used the 500 line $\mathrm{mm}^{-1}$ grating with a $1^{\prime \prime}$ slit width, resulting in an $\sim 3.6 \AA$ FWHM spectral resolution over a wavelength range of 3400-6100 A. These were reduced using standard IRAF routines and fluxcalibrated using the standard stars BD+284211 and Feige 34.

We obtained a second data set on UT 2010 February 8 with the Low Resolution Imaging Spectrometer (LRIS; Oke et al. $1995)$ on the Keck I telescope. We took these observations in long-slit mode using the blue side of the spectrograph with the atmospheric dispersion corrector. We used the 600 line $\mathrm{mm}^{-1} / 4000 \AA$ grism in combination with the D560 dichroic and a slit width of $1^{\prime \prime}$; the resulting data have a spectral resolution of $\approx 4.1 \AA$ FWHM. We obtained two exposures of $900 \mathrm{~s}$ each.

The Keck data were reduced using the onedspec package in IRAF. Overscan regions were used to subtract amplifier bias. The internal flat field was used to remove pixel-to-pixel sensitivity variations; small amplitude $(\approx 0.5 \%)$, large-scale $(\sim 100 \AA)$ residuals are present in the final flat-field. Cosmic rays were identified using the L.-A. Cosmic routine of van Dokkum (2001). The flat-fielded exposures were averaged; pixels flagged as cosmic rays were excluded from the averaging.

We extracted the one-dimensional spectrum and applied wavelength calibrations derived from arc lamp spectra. We also derived and applied an instrumental response calibration derived from the spectrophotometric standard star G 193-74 (spectral type DC); we make no attempt at absolute spectrophotometry. The resulting spectra, shown in Figure 6, have similar signal-tonoise ratios of $\approx 230$ per resolution element.

\subsection{Spectral Modeling}

One major difficulty in determining physical parameters of SDSS J1036+6522 was that tools for determining $T_{\text {eff }}$ and $\log g$ for strongly magnetic, carbon-helium mixed atmospheres simply did not exist. Therefore, one of us (P.D.) set out to develop model atmospheres appropriate for analyzing this star.

\subsubsection{Line Splitting}

In the presence of a weak magnetic field, typically up to a few tesla $\left(1 T=10^{4} \mathrm{G}\right)$, atoms can be described in the Zeeman regime. In that regime, an external magnetic field can be treated as a small perturbation to the Hamiltonian and the energy level properties can be obtained from first order perturbation theory. Assuming that the atom is described by $L-S$ coupling (a decent approximation for light atoms), $J$ and $m_{J}$ are good quantum numbers. In the presence of a weak magnetic field, an atomic level with total angular momentum $J$ splits into $2 J+1$ levels with magnetic quantum number $m_{J}=-J, \ldots,+J$. In that regime, the position of all magnetically shifted transitions between an upper and a lower level can easily be computed using the selection rule $(\Delta m=0, \pm 1)$ and $v_{u l}=\left(E_{u}-E_{l}\right) / h$, where $E_{i}=E_{i 0}+\left(e \hbar / 2 m_{e} c\right) g_{i} B m_{J}, B$ is the field strength, $E_{i 0}$ is the unperturbed energy of level $i$ and $g_{i}$ is the Lande factor of level $i$ (for recent applications in WD stars, see Farihi et al. 2011; Zuckerman et al. 2011).

For stronger magnetic fields able to produce splitting on a $J$-level that is comparable with the energy separation between the different $J$-levels of a term, $L$ and $S$ decouple and $J$ is no longer a good quantum number. When this happens, the perturbation theory (Zeeman effect) can no longer be applied. In this regime, called the Paschen-Back (PB) effect regime, the energy levels have to be found by the diagonalization of the total Hamiltonian $H=H_{0}+H_{B}$ where $H_{0}$ is the unperturbed Hamiltonian and $H_{B}=e \hbar / 2 m c(\mathbf{L}+2 \mathbf{S}) \cdot \mathbf{B}$.

Although the PB effect was successfully interpreted within the framework of quantum mechanics long ago (e.g., see classic textbooks such as Condon \& Shortley 1935), very few astronomical applications are found in non-degenerate stars. The main reason is that fields found in main sequence stars are not large enough to push most lines out of the Zeeman regime (sunspot field strengths are typically between 1 and $3 \mathrm{kG}$, while there are cases of a few Ap stars with field up to $30 \mathrm{kG}$ ), so only when the fine-structure levels of a term have abnormally small separation must the partial PB regime be taken into account. For such weak fields (by WD standards) the effect was only evidenced through the very small asymmetry that it induces in the lines profiles (Mathys 1990). Line profile calculations including the PB effect have been recently incorporated in stellar models only for a few sensitive lines in Ap stars (Fe II multiplet 


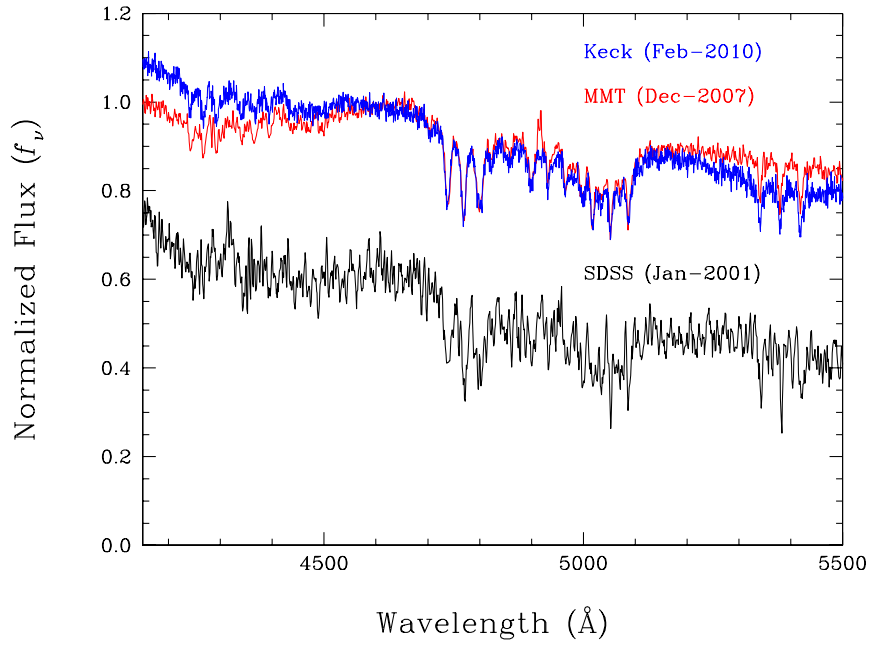

Figure 6. Normalized spectroscopic data for SDSS J1036+6522. The small differences between the continuum level of MMT (red) and Keck (blue) data are due to errors in flux calibration. A three-point average smoothing window and a downward shift has been applied on the SDSS data for clarity. The majority of the visible features are $\mathrm{C}_{\mathrm{I}}$ with magnetic splitting.

74 and Li $\lambda 6707$ doublet, see Stift et al. 2008; Kochukhov 2008) and the Sun (molecular lines and He I $\lambda 10830$, see Asensio Ramos et al. 2005; Berdyugina et al. 2006; Socas-Navarro et al. 2005).

Magnetic fields strong enough to push the lines into the PB regime are commonly found in WD stars (see the latest review by Jordan 2009 and references therein), yet since the vast majority of known magnetic WD stars only show hydrogen line splitting, only hydrogen-rich magnetic model atmospheres have been developed in detail to this date. However, the population of magnetic WDs with heavy element features is growing rapidly thanks to the work on DQ (see Schmidt et al. 1995), hot DQ (Dufour et al. 2009b) and DZ WDs (i.e., LHS 2534 and G165-7; Reid et al. 2001; Dufour et al. 2006). Accurate determination of the atmospheric parameters for such stars requires the development of a new generation of magnetic model atmospheres that include various field geometries, radiative transfer for all Stokes components, as well as splitting in the $\mathrm{PB}$ regime. Although such models are not available yet, it is still possible to obtain acceptable results with a few reasonable approximations.

\subsubsection{Models and Synthetic Spectra}

As mentioned above, detailed magnetic model atmospheres appropriate for WD stars showing heavy elements are currently unavailable. Nevertheless, it is possible to obtain a good first order approximation with some reasonable assumptions. We first assume that the thermodynamic structure is not affected by the magnetic field, a conservative approximation for fields of a few MG according to Jordan (1992). We also calculate our synthetic spectrum using normal radiative transfer, though unfortunately it is currently unknown to what extent the use of formal radiative transfer equations for all Stokes parameters would affect our results. Finally, we simply assume a uniform magnetic field strength over the surface of the stars (which seems to be indicated by our observational data, in particular the sharp and well separated components of the magnetically split lines in Figure 8). Future work, which should address all these issues, will be presented in due time. Equipped with this

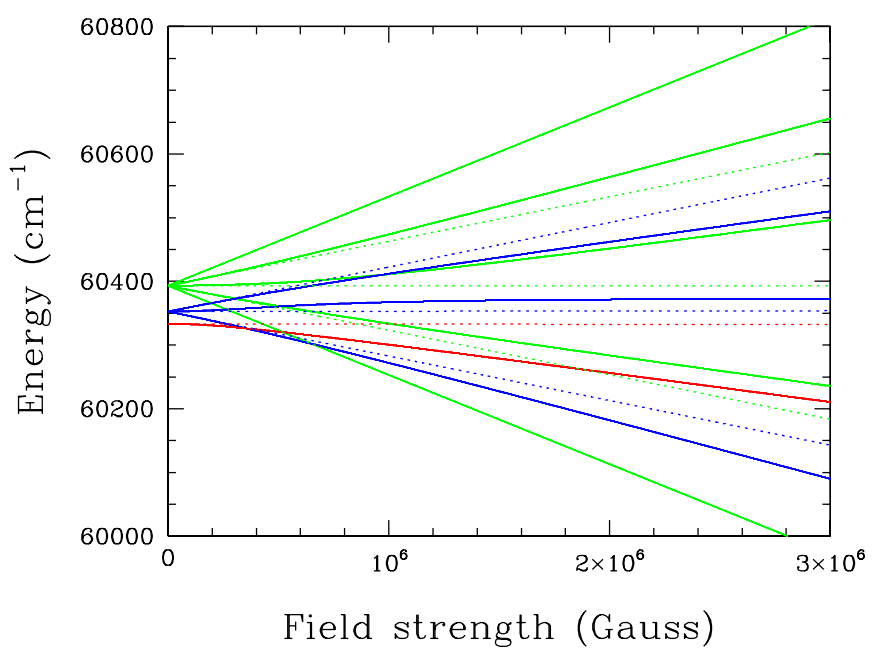

Figure 7. Magnetic splittings of the three lower sub-levels of the ${ }^{3} P$ term of the $\mathrm{C}$ I multiplet near $4775 \AA$. Dotted lines show the energy level as a function of magnetic field strength assuming Zeeman splitting while the full line is the result of the calculations in the Paschen-Back regime (see text).

simple theoretical framework, we next calculate a grid of model atmospheres appropriate for warm DQ stars.

Our LTE model atmosphere code is similar to that presented in Dufour et al. (2005) and Dufour et al. (2008a) except that we use absorption line data from the Vienna Atomic Line Database (VALD). Our model grid covers a range of effective temperature from $T_{\text {eff }}=11,500-19,000 \mathrm{~K}$ in steps of $500 \mathrm{~K}, \log g=8.0-9.5$ in steps of $0.5 \mathrm{dex}$, and from $\log (\mathrm{C} / \mathrm{He})=0.0$ to -3.5 in steps of 0.5 dex.

Detailed synthetic fluxes are then obtained from these atmospheric structures by first including line splitting in the Zeeman regime for a magnetic field of $3 \mathrm{MG}$. This value is obtained from measuring the separation of the $\pi(\Delta m=0)$ and $\sigma(\Delta m= \pm 1)$ components of the well isolated C I $5380 \AA$ triplet, shown in Figure 6 . Note that the separation between the components for this transition are not different in the PB regime. To estimate the error, we also calculated a few supplementary model with magnetic field slightly different than 3 MG. From this experiment, we find that varying the field by more than $0.2 \mathrm{MG}$ changes the line positions sufficiently to be detected by visual inspection. Hence, we adopt a field strength of $3.0 \pm 0.2 \mathrm{MG}$.

While a $3 \mathrm{MG}$ magnetic field is appropriate to reproduce the observed splitting in the $\sim 5380 \AA$ features, it fails to reproduce the position of the carbon feature between $\sim 4700-5000 \AA$ (see the synthetic spectra calculated under the Zeeman regime assumption in Figure 8). As explained above, the most likely explanation is that the magnetic field is sufficiently strong to push some of the lines in the PB regime where the position and strength of spectral features differ to that predicted by simple Zeeman calculations.

Indeed, as can be observed in Figure 7, the position of the energy levels produced by a 3 MG magnetic field can sometimes be significantly different in the Zeeman and PB regime. Under such circumstances, the energy levels and line strength values due to the magnetic field perturbation must thus be obtained by the diagonalization of a set of matrices for each of the transitions included in our synthetic spectra. We thus proceeded in calculating the line strength and positions of all the components of magnetically split $\mathrm{C}_{\mathrm{I}}$ lines in the $\mathrm{PB}$ regime (the relevant equations are described in detail in Section 3.4 of the monograph by Landi Degl'Innocenti \& Landolfi 2004). 


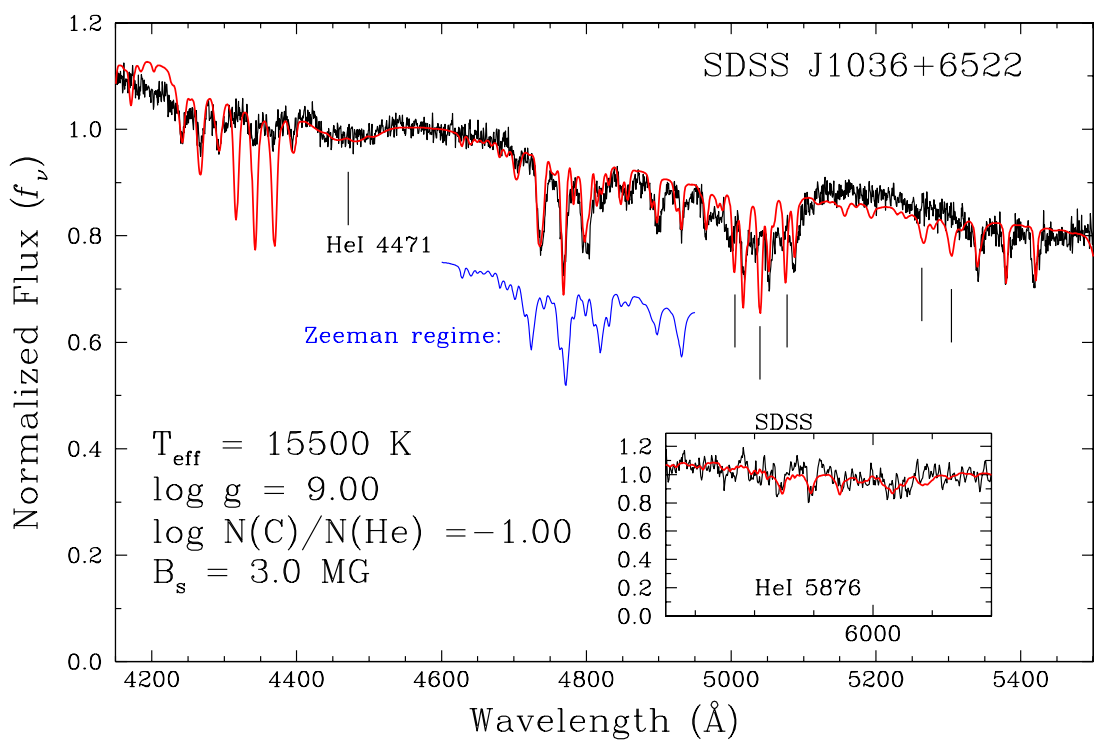

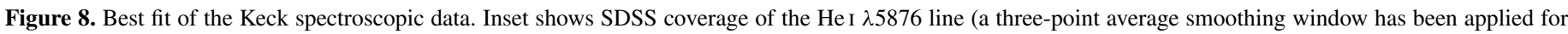

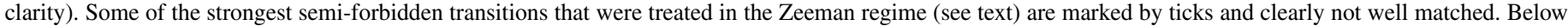

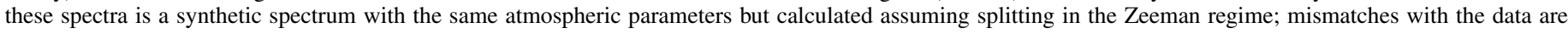
obvious.

NIST Atomic Spectra Database energy levels were used as input for the PB calculations, though the line strengths of each transition are obtained by re-normalizing appropriately the sum of all the $\log g f$ of a given multiplet taken from the VALD database.

Our final grid of synthetic spectra is calculated with a mean magnetic field of $3 \mathrm{MG}$ based on the separation of the $5380 \AA$ lines, which are transitions between ${ }^{1} P$ terms that are correctly described with the Zeeman regime. Since the positions of the He I lines are correctly reproduced in the Zeeman regime, it was unnecessary to perform the lengthy PB calculation for helium for this exploratory analysis.

Finally, we note that our $C_{\text {I }}$ line list contained a few semiforbidden transitions that violate the selection rules $(\Delta S=0$ and/or $\Delta L= \pm 1,0)$. The PB formalism described above cannot be used for these lines and a more general approach needs to be implemented in that case. Unfortunately, the relevant data necessary for such calculations are currently unavailable. We thus simply modeled those transitions assuming normal Zeeman splitting patterns, an approximation that does not correctly represent the positions and strength of such lines (see, for example, tick marks in Figure 8).

\subsubsection{Fitting Technique, Results, and Discussion}

We attempted to fit the spectra using the usual non-linear leastsquare routines commonly used in spectral fitting, but these led to regions of parameter space that made little sense. For example, a fit might converge successfully on a minimum $\chi^{2}$, but visual inspection would reveal obvious shortcomings such as not reproducing the $4471 \AA$ He I dip, not matching the slope, or matching the strengths and positions of one group of lines while another set was poorly fit. It is likely that the models do not perfectly reproduce reality because of the numerous approximations, and some badly reproduced regions (such as the group of $\mathrm{C}_{\mathrm{I}}$ lines near $4340 \AA$ that are always too strong, the semi-forbidden transitions, etc.) are simply pulling the solution away from realistic values. Even when we fixed $\log g$ and $T_{\text {eff }}$, the carbon abundance would wander to unrealistic values that resulted in obvious visual mismatches. We therefore resorted to visual inspection for selecting the best model.

Another difficulty is the strong group of C I lines near $4340 \AA$. Choices of parameters that fit this complex well result in very poor fits for the rest of the spectrum; there is no combination of parameters in our model grid that can achieve a satisfactory fit over all spectral region simultaneously. This could imply that our numerous approximations affect the temperature structure in such a way to poorly reproduce this complex or perhaps that the $\log g f$ values for these lines are incorrect.

Our visual inspection therefore starts with the $\mathrm{C}$ II lines near $4267 \AA$. Since this is an ionized feature, it provides decent constraints on $T_{\text {eff }}$. We then use the broad He I $4471 \AA$ dip as a main indicator of carbon abundances, as this dip proves quite sensitive to $\log (\mathrm{C} / \mathrm{He})$. In parallel, the MMT and SDSS spectra include the He I $5876 \AA$ line, which we used as a confirmation of the abundance. This line also allows some of the coolest solutions to be rejected.

Our best solution, shown in Figure 8 , has $T_{\text {eff }}=15,500 \mathrm{~K}$, $\log g=9$, and $\log (\mathrm{C} / \mathrm{He})=-1.0$. The solutions are degenerate; similar quality solutions can be found by compensating a downward change in $T_{\text {eff }}$ with downward changes in $\log g$ and $\log (\mathrm{C} / \mathrm{He})$. We estimate our correlated uncertainties in these parameters as follows. A $\sim 1000 \mathrm{~K}$ change in our best solution's $T_{\text {eff }}$ resulted in a $\sim 0.5$ dex change in $\log (\mathrm{C} / \mathrm{He})$, with a lower $T_{\text {eff }}$ resulting in less carbon and vice versa. A change in $\log g$ has little effect on the carbon abundance as long as we stay close to $\log g=9.0$; at $15,500 \mathrm{~K}$ and $\log g=8.5, \log (\mathrm{C} / \mathrm{He})$ is only 0.2 dex higher. At $\log g=8.0$, the carbon abundance increases by about 0.7 dex as compared to $\log g=9$. Even though $\log g=9$ provides the most appealing solution visually, given all the approximations and the preliminary nature of our calculations, our value of $\log g$ should not be considered a precision measurement. Much more work is needed before we can confidently determine the masse of this object.

Carbon-rich solutions, favored for hot DQs, do not fit well. However, we can impose some useful additional constraints on these parameters. For instance, we compared the SDSS photometry of SDSS J1036+6522 with synthetic photometry calculated 


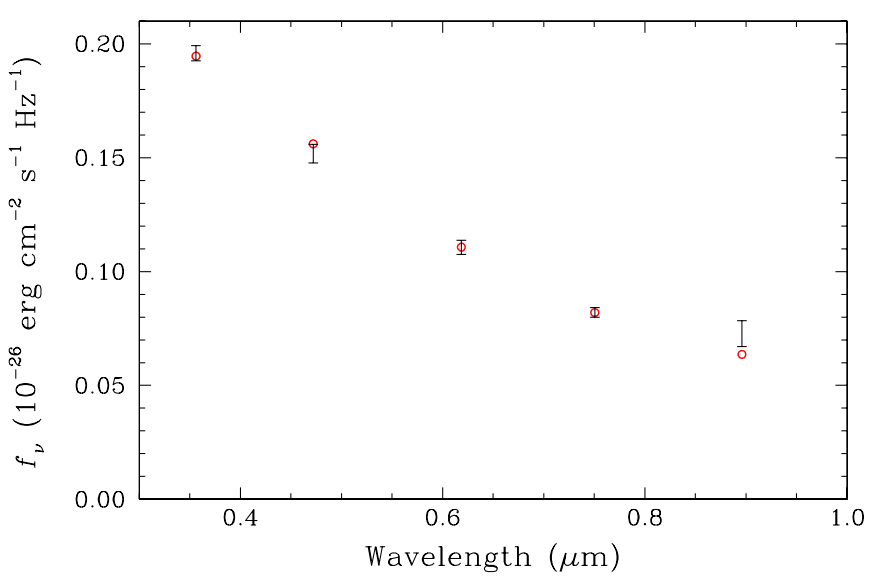

Figure 9. SDSS photometric observations in the ugriz bands (error bars) and average model fluxes for our favored atmospheric parameters (circles; red in the online version of the figure; see text). The photometric observations have been corrected for extinction as prescribed by the reddening maps of Schlegel et al. (1998).

(A color version of this figure is available in the online journal.)

by folding the model spectra through filter response curves. The photometric data (Figure 9) seem to favor a solution in the $15,000-16,000 \mathrm{~K}$ range, a temperature range which is corroborated by the He I $5876 \AA$ line, although the unknown reddening makes this a bit uncertain. This conclusion also supports the qualitative best matching model above.

\subsection{What is SDSS J1036+6522?}

Based on the spectroscopic and photometric analysis, SDSS J1036+6522 seems to be an intermediate object between the classical, cool DQ stars $\left(T_{\text {eff }} \lesssim 10,000 \mathrm{~K}\right.$; e.g., Dufour et al. $2005)$ and hot DQ stars $\left(18,000 \mathrm{~K} \lesssim T_{\text {eff }} \lesssim 24,000 \mathrm{~K}\right.$; Dufour et al. 2008a, 2007). Its high carbon abundance also seems to make it akin to the second sequence of carbon-rich cool DQs (Dufour et al. 2005; Koester \& Knist 2006) and two DQs with similar temperature, G35 - 26 (Thejll et al. 1990) and G227 - 5 (Wegner \& Koester 1985). In addition, the high magnetic field of the star also appears to be a typical characteristic of many of the hot DQs (Dufour et al. 2009b).

Although the high apparent surface gravity should be considered preliminary, a growing body of evidence suggests that hot DQs and the carbon-rich sequence of cool DQs are massive WDs. This evidence includes unpublished parallaxes of G35-26 and G227-5 (Liebert et al. 2003; C. C. Dahn 2003, private communication); the high mass of the carbon- and oxygenrich post-AGB star H1504+65 (Werner et al. 2004); the presence of a hot DQ in the open star cluster Messier 35, in which the DA cluster member WDs are all massive (Williams et al. 2006, 2009); and improved spectroscopic observations and model atmospheres of hot DQs, which favor high surface gravities (P. Dufour et al., in preparation).

We note however that these calculations are done with classical approximations for the Stark broadening of C I lines, so the high gravity solution should be considered highly uncertain. State-of-the-art Stark broadening parameters for C I similar to that calculated for C II in Dufour et al. (2011b) are unfortunately not available yet.

In summary, SDSS J1036+6522 is most probably a cooler version of SDSS J1402+3818 and SDSS J0005-1002 (see Dufour et al. 2009b) and/or a hotter version of G227-5 and G35-26. SDSS J1036+6522 could thus be a transition object between the coolest carbon-rich hot DQs and the hottest, cool heliumrich DQ WDs. There is thus growing evidence that this class of WDs indeed forms a distinct and fairly continuous evolutionary sequence of massive hydrogen- and helium-deficient stars, as proposed by Dufour et al. (2008a). Further analysis on this type of objects will require improved models including various magnetic field geometries, solving the radiative transfer equation for all Stokes parameters (spectropolarimetric observations would be especially useful to test the eventual predictions from such models), and inclusion of state-of-the-art Stark broadening parameters for $\mathrm{C}$ I once available.

\section{DISCUSSION}

The detection of variability in SDSS J1036+6522 increases yet again the number of known DQVs. However, the spectroscopic analysis raises the question of whether or not SDSS J1036+6522 belongs in the same class as the other known DQVs.

There are some crucial differences between SDSS J1036+6522 and the hot DQVs, first and foremost being $T_{\text {eff }}$, with SDSS J1036+6522 being significantly cooler than the other DQVs, as discussed above. Second, the atmospheric composition of SDSS J1036+6522 is different than both the hot and cool DQs. Hot DQs are carbon-dominated, with $\log (\mathrm{C} / \mathrm{He}) \geqslant 0$, and He usually undetected (Dufour et al. 2008a). In the "secondsequence" cool DQs, $\log (\mathrm{C} / \mathrm{He}) \lesssim-3$ (Koester \& Knist 2006; Dufour et al. 2005). Again, SDSS J1036+6522 is intermediate, with $\log (\mathrm{C} / \mathrm{He}) \approx-1$. This observation furthers the hypothesis that SDSS J1036+6522 is a transition object between hot and cool DQs.

We now explore the interpretations and implications of this conclusion.

\subsection{What is the Cause of the Variability?}

From an observational standpoint, the photometric variability of SDSS J1036+6522 is very similar to the other known DQVs. These similar characteristics include the period and amplitude of the photometric variability, the dominance of a single frequency in the DFT, and the stability of the frequency and phase.

Based on the time-series photometry, SDSS J1036+6522 looks and acts like the other members of the DQV class of objects. Naively combining this information with the spectroscopic evidence that this star belongs to the same evolutionary sequence and object class as the hot DQVs, we would come to the conclusion that SDSS J1036+6522 is a typical DQV and is variable for the same reason as the others, whatever that reason may be.

The difficulties arise when we attempt to explain the origin of the photometric variations. As described in the introduction, the preferred explanation for the variability in DQVs has been nonradial pulsations. Even though there are differing physical mechanisms and different predicted instability strips for pulsations in DQVs, SDSS J1036+6522 is far cooler than any of these predictions (though we note that these models do not include a significant magnetic field). Moreover, if our atmospheric analysis is correct, the atmosphere of SDSS J1036+6522 is helium dominated and $\sim 10^{4} \mathrm{~K}$ cooler than the coolest observed DBV (e.g., Nitta et al. 2009).

Montgomery et al. (2008) hypothesize that the periodic photometric variability could be due to an accreting system akin to high-accretion states of AM CVn systems. This speculation was based solely on the observed pulse shape of SDSS J1426+5752. 
This hypothesis now appears highly unlikely. High signalto-noise spectra of DQVs show no signs of accretion (e.g., Dufour et al. 2009b, 2011a), radial-velocity variations have not been detected with cataclysmic-variable-like periods (Green et al. 2009, although orbital periods commensurate with the photometric period have not yet been ruled out), and no lowaccretion state systems analogues have been identified. Given that SDSS J1036+6522 has a sinusoidal pulse shape, we find no evidence that SDSS J1036+6522 is an interacting binary system.

Another possibility, discussed by Dufour et al. (2008b), is that this star is a WD analogue of rapidly oscillating Ap (roAp) stars (e.g., Kurtz 1982). Given the presence of a strong magnetic field, this oblique pulsator model seems to be a distinct possibility.

It is also plausible that SDSS J1036+6522 is a rapidly rotating $\mathrm{WD}$, with the photometric variations due to the presence of a persistent spot, although several arguments could be made against this interpretation. Accreting magnetic objects such as AM Her systems show photometric modulation due in part to the rotation of the WD carrying the accretion column in and out of view, but SDSS J1036+6522 shows no evidence of accretion. In addition, the measured rotation periods of single magnetic WDs are on the order of hours and days to centuries (Schmidt \& Norsworthy 1991), significantly longer than the photometric period of SDSS J1036+6522. As the measured rotation rates of other classes of isolated pulsating WDs are also on the order of hours to days (e.g., Winget \& Kepler 2008; Fontaine \& Brassard 2008 , and references therein), the rotation hypothesis would require SDSS J1036+6522 to be an outlier among single WDs. Time-resolved spectropolarimetry may be able to determine if SDSS J1036+6522 is rapidly rotating.

At present, it is unclear what the cause of the photometric variability in SDSS J1036+6522 is, or even if the variability has the same cause as for the hot DQVs.

\subsection{Magnetic Fields and Pulse Shapes}

Typically, large amplitude DAV and DBV pulsators tend to have relatively non-linear (non-sinusoidal) pulse shapes, while low-amplitude pulsators tend to be more sinusoidal. This relation does not appear to hold among the known DQVs with sufficient data to calculate pulse shapes. In the prototype DQV SDSS J1426+5752, the amplitude of the first harmonic (6.7 $\mathrm{mma})$ is less than half that of the fundamental $(17.5 \mathrm{mma}$; Montgomery et al. 2008), while in the much lower amplitude DQV SDSS J2200-0741, the first harmonic is similar in amplitude to the fundamental frequency at $\approx 7 \mathrm{mma}$ (Barlow et al. 2008; Dufour et al. 2009a). These significant harmonics and their phases result in decidedly non-sinusoidal, boxy pulse shapes with long brightness maxima and short, narrow minima, the opposite to the pulse shapes found in the high amplitude DAVs and DBVs (Montgomery et al. 2008). However, the similar-amplitude DQV SDSS J2348-0943 ( $\approx 8$ mma; Barlow et al. 2008; Dufour et al. 2009a) is sinusoidal, and the lowamplitude DQV SDSS 1337-0026 ( $\approx 4$ mma; Dunlap et al. 2010) has a very non-sinusoidal pulse shape.

Further, the three DQVs with non-sinusoidal pulse shapes all have detectable magnetic fields, while the sinusoidal SDSS J2348-0943 does not. This has led to the suggestion by Green et al. (2009) that the magnetic field may be responsible for the non-linearities observed in many DQVs.

The folded pulse shape of SDSS J1036+6522 appears to be sinusoidal. This qualitative statement is reinforced quantitatively by the lack of any observed harmonics in the DFT. As SDSS J1036+6522 is strongly magnetic yet has a sinu- soidal pulse shape (within the limits of existing data), it is clear that strong magnetic fields in DQVs do not always cause boxy pulse shapes. Strong magnetic fields in SDSS J1337-0026, SDSS J1426+5752 and SDSS J2200-0741 may well be the cause of their unique pulse shapes, but SDSS J1036+6522 shows that a sinusoidal pulse shape in a DQV does not indicate the absence of a strong magnetic field, at least assuming that the mechanism behind the variability is the same for SDSS J1036+6522 and the hotter DQVs.

\subsection{Non-variable Hot DQs}

Montgomery et al. (2008) claim that variability was not detected in several hot DQs, as well as that those stars would not be expected to vary given their $T_{\text {eff }}$ and $\log g$ and given predictions for an instability strip. In light of the low amplitude of variability in SDSS J1036+6522 and SDSS J1337-0026 (Dunlap et al. 2010) and the typical detection threshold of $\sim 5-10 \mathrm{mma}$ in the data obtained for Montgomery et al. (2008), we feel compelled to mention that many of the stars not observed to vary could in fact be variable at the same level as SDSS J1036+6522. Photometric precision of equal to or better than 1 mma would be required to rule out SDSS J1036+6522like variability.

Therefore, we feel that the claims of non-detection of variability in Montgomery et al. (2008) should be viewed with the caveat that these limits are not very significant. As Dufour et al. (2011a) detect UV variability in four out of five target hot DQs, it could be that most or all hot DQs are in fact DQVs. A reanalysis of the time-series data for stars not observed to vary is underway, and more stringent limits on non-detections of variability will be presented in a forthcoming paper. These limits will also be crucial for testing competing models for these stars, such as the different proposed instability strips discussed in the introduction.

\section{CONCLUSIONS}

In summary, we present spectroscopy and time-series photometry of the WD SDSS J1036+6522. Our analyses lead us to the following conclusions:

1. SDSS J1036+6522 is photometrically variable with a period of $1115.64751(67) \mathrm{s}$ and an amplitude of $0.442 \% \pm 0.024 \%$. The period, amplitude, and phase of the modulations are constant within measurement errors over at least a sixteenmonth interval.

2. SDSS J1036+6522 has an effective temperature and carbon abundance located between the hot and cool DQs, suggesting that it is a transition object between those two populations of WDs.

3. The star is magnetic, with a field strength of $3.0 \pm 0.2 \mathrm{MG}$.

4. The lack of observed harmonics suggest that the photometric modulation is nearly sinusoidal. If the cause of the photometric modulation is the same mechanism as in the hot DQVs, then the absence or strength of the star's magnetic field is not necessarily correlated with the pulse shape.

5. The mechanism of the photometric modulation in SDSS J1036+6522 is unclear. There is no evidence for accretion, the effective temperature is significantly cooler than pulsation models predict, and the photometric period is significantly shorter than the typical isolated WD rotational period.

6. Photometric variability is common among hot DQ WDs, but the low optical amplitudes of many known DQVs imply 
that non-detections of variability in any hot DQ are only significant if photometric precision is high $(\sim 0.1 \%)$.

K.A.W. is grateful for the financial support of National Science Foundation award AST-0602288. D.E.W., M.H.M., J.J.H., R.E.F., and K.I.W. acknowledge the support of the NSF under grant AST-0909107 and the Norman Hackerman Advanced Research Program under grant 003658-0252-2009. We thank E. Landi Delgl'Innocenti for useful discussions and his help in modeling lines in the Paschen-Back regime. P.D. is a CRAQ postdoctoral fellow. We acknowledge the use of the VALD Database (Piskunov et al. 1995; Ryabchikova et al. 2008; Kupka et al. 1999, 2000). We are very grateful for the technical support provided by D. Chandler during the photometric observations and for the many fine improvements to the Struve telescope and telescope control spearheaded by J. Kuehne and the McDonald Observatory staff. Ron Leck and R. E. Nather were indispensable in upgrading the instrumental software during these runs. We also appreciate the comments and insight of the anonymous referee.

Facilities: Struve (Argos), Keck:I (LRIS), MMT (Blue Channel)

\section{REFERENCES}

Abazajian, K. N., Adelman-McCarthy, J. K., Agüeros, M. A., et al. 2009, ApJS, 182,543

Althaus, L. G., García-Berro, E., Córsico, A. H., Miller Bertolami, M. M., \& Romero, A. D. 2009, ApJL, 693, L23

Asensio Ramos, A., Trujillo Bueno, J., \& Collados, M. 2005, ApJL, 623, L57

Barlow, B. N., Dunlap, B. H., Rosen, R., \& Clemens, J. C. 2008, ApJL, 688, L95

Berdyugina, S. V., Fluri, D. M., Ramelli, R., et al. 2006, ApJL, 649, L49

Condon, E. U., \& Shortley, G. H. 1935, The Theory of Atomic Spectra (Cambridge: Cambridge Univ. Press), 460

Córsico, A. H., Romero, A. D., Althaus, L. G., \& García-Berro, E. 2009, A\&A, 506,835

Dufour, P., Béland, S., Fontaine, G., Chayer, P., \& Bergeron, P. 2011a, ApJL, 733, L19

Dufour, P., Ben Nessib, N., Sahal-Bréchot, S., \& Dimitrijević, M. S. 2011b, BaltA, 20, 511

Dufour, P., Bergeron, P., \& Fontaine, G. 2005, ApJ, 627, 404

Dufour, P., Bergeron, P., Schmidt, G. D., et al. 2006, ApJ, 651, 1112

Dufour, P., Fontaine, G., Liebert, J., Schmidt, G. D., \& Behara, N. 2008a, ApJ, 683, 978

Dufour, P., Fontaine, G., Liebert, J., Williams, K., \& Lai, D. K. 2008b, ApJL, 683, L167

Dufour, P., Green, E. M., Fontaine, G., et al. 2009a, ApJ, 703, 240

Dufour, P., Liebert, J., Fontaine, G., \& Behara, N. 2007, Natur, 450, 522

Dufour, P., Liebert, J., Swift, B., Fontaine, G., \& Sukhbold, T. 2009b, JPhCS, 172,012012

Dunlap, B. H., Barlow, B. N., \& Clemens, J. C. 2010, ApJL, 720, L159

Dupuis, J., Fontaine, G., Pelletier, C., \& Wesemael, F. 1992, ApJS, 82, 505

Dupuis, J., Fontaine, G., Pelletier, C., \& Wesemael, F. 1993, ApJS, 84, 73

Eastman, J., Siverd, R., \& Gaudi, B. S. 2010, PASP, 122, 935

Eisenstein, D. J., Liebert, J., Harris, H. C., et al. 2006, ApJS, 167, 40

Farihi, J., Dufour, P., Napiwotzki, R., \& Koester, D. 2011, MNRAS, 413, 2559

Farihi, J., Jura, M., \& Zuckerman, B. 2009, ApJ, 694, 805
Fontaine, G., \& Brassard, P. 2008, PASP, 120, 1043

Fontaine, G., Brassard, P., \& Bergeron, P. 2001, PASP, 113, 409

Fontaine, G., Brassard, P., \& Dufour, P. 2008, A\&A, 483, L1

Garcia-Berro, E., Ritossa, C., \& Iben, I., Jr. 1997, ApJ, 485, 765

Green, E. M., Dufour, P., Fontaine, G., \& Brassard, P. 2009, ApJ, 702, 1593

Hansen, B. M. S. 1999, ApJ, 520, 680

Hansen, B. M. S., Richer, H. B., Fahlman, G. G., et al. 2004, ApJS, 155, 551

Iben, I., Jr., Kaler, J. B., Truran, J. W., \& Renzini, A. 1983, ApJ, 264, 605

Jordan, S. 1992, A\&A, 265, 570

Jordan, S. 2009, in IAU Symp. 259, Cosmic Magnetic Fields: From Planets, to Stars and Galaxies, ed. K. G. Strassmeier, A. G. Kosovichev, \& J. E. Beckman (Cambridge: Cambridge Univ. Press), 369

Jura, M. 2003, ApJL, 584, L91

Kepler, S. O. 1993, BaltA, 2, 515

Kochukhov, O. 2008, A\&A, 483, 557

Koester, D. 1976, A\&A, 52, 415

Koester, D., \& Knist, S. 2006, A\&A, 454, 951

Kupka, F., Piskunov, N., Ryabchikova, T. A., Stempels, H. C., \& Weiss, W. W. 1999, A\&AS, 138, 119

Kupka, F. G., Ryabchikova, T. A., Piskunov, N. E., Stempels, H. C., \& Weiss, W. W. 2000, BaltA, 9, 590

Kurtz, D. W. 1982, MNRAS, 200, 807

Landi Degl'Innocenti, E., \& Landolfi, M. 2004, Polarization in Spectral Lines (Astrophysics and Space Science Library, Vol. 307; Dordrecht: Kluwer)

Liebert, J., Harris, H. C., Dahn, C. C., et al. 2003, AJ, 126, 2521

Mathys, G. 1990, A\&A, 232, 151

Monet, D. G., Levine, S. E., Canzian, B., et al. 2003, AJ, 125, 984

Montgomery, M. H., Williams, K. A., Winget, D. E., et al. 2008, ApJL, 678, L51

Mullally, F., Thompson, S. E., Castanheira, B. G., et al. 2005, ApJ, 625, 966

Mullally, F., Winget, D. E., Degennaro, S., et al. 2008, ApJ, 676, 573

Nather, R. E., \& Mukadam, A. S. 2004, ApJ, 605, 846

Nitta, A., Kleinman, S. J., Krzesinski, J., et al. 2009, ApJ, 690, 560

Oke, J. B., Cohen, J. G., Carr, M., et al. 1995, PASP, 107, 375

Piskunov, N. E., Kupka, F., Ryabchikova, T. A., Weiss, W. W., \& Jeffery, C. S. 1995, A\&AS, 112, 525

Reid, I. N., Liebert, J., \& Schmidt, G. D. 2001, ApJL, 550, L61

Ryabchikova, T., Kildiyarova, R., Piskunov, N., et al. 2008, JPhCS, 130, 012017

Scargle, J. D. 1982, ApJ, 263, 835

Schlegel, D. J., Finkbeiner, D. P., \& Davis, M. 1998, ApJ, 500, 525

Schmidt, G. D., Bergeron, P., \& Fegley, B. 1995, ApJ, 443, 274

Schmidt, G. D., Harris, H. C., Liebert, J., et al. 2003, ApJ, 595, 1101

Schmidt, G. D., \& Norsworthy, J. E. 1991, ApJ, 366, 270

Socas-Navarro, H., Trujillo Bueno, J., \& Landi Degl'Innocenti, E. 2005, ApJS, 160,312

Stift, M. J., Leone, F., \& Landi Degl'Innocenti, E. 2008, MNRAS, 385, 1813

Stumpff, P. 1980, A\&AS, 41, 1

Thejll, P., Shipman, H. L., MacDonald, J., \& Macfarland, W. M. 1990, ApJ, 361,197

Tremblay, P.-E., \& Bergeron, P. 2008, ApJ, 672, 1144

van Dokkum, P. G. 2001, PASP, 113, 1420

von Hippel, T. 2005, ApJ, 622, 565

Wegner, G., \& Koester, D. 1985, ApJ, 288, 746

Weidemann, V. 1990, ARA\&A, 28, 103

Werner, K., Rauch, T., Barstow, M. A., \& Kruk, J. W. 2004, A\&A, 421, 1169

Williams, K. A., Bolte, M., \& Koester, D. 2009, ApJ, 693, 355

Williams, K. A., Liebert, J., Bolte, M., \& Hanson, R. B. 2006, ApJL, 643, L127

Winget, D. E., Hansen, C. J., Liebert, J., et al. 1987, ApJL, 315, L77

Winget, D. E., \& Kepler, S. O. 2008, ARA\&A, 46, 157

Winget, D. E., Robinson, E. L., Nather, R. E., Kepler, S. O., \& Odonoghue, D. 1985, ApJ, 292, 606

Zuckerman, B., Koester, D., Dufour, P., et al. 2011, ApJ, 739, 101

Zuckerman, B., Koester, D., Melis, C., Hansen, B. M., \& Jura, M. 2007, ApJ, 671,872

Zuckerman, B., Koester, D., Reid, I. N., \& Hünsch, M. 2003, ApJ, 596, 477 